\title{
Expanding preconception carrier screening for the Jewish population using high throughput microfluidics technology and next generation sequencing
}

Moran Gal ${ }^{1}$, Khen Khermesh ${ }^{1}$, Michal Barak', Min Lin², Hadas Lahat ${ }^{3}$, Haike Reznik Wolf ${ }^{3}$, Michael Lin², Elon $\operatorname{Pras}^{3,4}$ and Erez Y. Levanon ${ }^{1 *}$

\begin{abstract}
Background: Genetic screening to identify carriers of autosomal recessive diseases has become an integral part of routine prenatal care. In spite of the rapid growth of known mutations, most current screening programs include only a small subset of these mutations, and are performed using diverse molecular techniques, which are generally labor-intensive and time consuming. We examine the implementation of the combined high-throughput technologies of specific target amplification and next generation sequencing (NGS), for expanding the carrier screening program in the Israeli Jewish population as a test case.

Methods: We compiled a panel of 370 germline mutations, causing 120 disorders, previously identified in affected Jewish individuals from different ethnicities. This mutation panel was simultaneously captured in 48 samples using a multiplex PCR-based microfluidics approach followed by NGS, thereby performing 17,760 individual assays in a single experiment.

Results: The sensitivity (measured with depth of at least 50x) and specificity of the target capture was 98 and $95 \%$ respectively, leaving minimal rate of inconclusive tests per sample tested. $97 \%$ of the targeted mutations present in the samples were correctly identified and validated.

Conclusion: Our methodology was shown to successfully combine multiplexing of target specific primers, samples indexing and NGS technology for population genetic screens. Moreover, it's relatively ease of use and flexibility of updating the targets screened, makes it highly suitable for clinical implementation. This protocol was demonstrated in pre-conceptional screening for pan-Jewish individuals, but can be applied to any other population or different sets of mutations.
\end{abstract}

Keywords: Carrier screening, Next generation sequencing, Microfluidics, Genetic testing, Jewish population

\section{Background}

Genetic screening to identify carriers of recessive diseases (also known as preconception, premarital, prenatal, or reproductive screening) aims to detect couples at risk for transmitting hereditary genetic diseases to their offspring. The purpose of such screening is to provide the prospective parents the opportunity to make informed

\footnotetext{
* Correspondence: erez.levanon@biu.ac.il

'The Mina and Everard Goodman Faculty of Life Sciences, Bar-llan University, Ramat Gan 52900, Israel

Full list of author information is available at the end of the article
}

decisions regarding their reproductive options or the use of early interventions (when available). From a public health perspective, carrier screening programs reduce the incidence of genetic diseases, and are cost-effective from medical expenditure aspect.

The introduction of NGS platforms in recent years enables DNA sequencing at an unprecedented yield and low cost. It is clear that this technology will become the method-of-choice for any clinical genetic testing program and it holds the potential of expanding the current screening programs for reproductive choices, in a similar 
manner that mass spectrometry has enabled screening of newborns for metabolites [1]. Despite the feasibility of sequencing entire exomes and genomes, such an approach is still not affordable or applicable for population screening, in which thousands of samples must be analyzed and unambiguous results must be obtained automatically in a timely manner. Thus, for the foreseeable future, a targeted re-sequencing method is still required. Moreover, the screening techniques chosen should be flexible and scalable, in order to keep up with the new genetic discoveries and rapid growth of mutation databases.

In this work we explored the feasibility of microfluidics technology for target capture, followed by NGS to expand carrier screening for reproductive purposes (Fig. 1). The Jewish population in Israel was chosen as a test case. Carrier screening in the Jewish population for reproductive purposes has been carried out for decades. Since the first preconception carrier screening program for Tay Sachs disease was introduced for Ashkenazi Jews in the 1970s [2], additional common mutations for other severely debilitating diseases in the Jewish population were identified, and have been introduced into screening panels [3-5]. As a result, to date, the birth of affected children with these diseases is rare, and the screening programs have essentially become the "standard of care", administered either premarital, before conception, or during the pregnancy.

An updated catalog of disease-causing mutations found in Jewish population contains more than 1000 different mutations responsible for hundreds disorders (http://server.goldenhelix.org/israeli/) [6]. Yet, currently in most centers in Israel, genetic testing is offered for only a limited subset of these ( $\sim 30$ diseases). The major limitations for introducing new mutations into screening panels are financial and technological constraints, such as current laboratory techniques, human labor and test execution time.

The official recommendations for genetic screening in Israel from the Israeli Society of Medical Genetics [7] and the Israeli Ministry of Health $(\mathrm{MOH})$ [8] (and its public funding) are largely based on the frequencies of the mutations in the population (i.e. testing is prioritized for more common mutations and avoided for the rarer ones), and the screening is performed in an ethnicdependent manner (i.e. different mutation panels for Ashkenazi Jews, Jews originating from North Africa, Jews from Iraq, etc.).

However, over the past decades, the inter-ethnic marriage rate has increased among the Jewish population in Israel [9], and thus, choosing the relevant set of genetic disorders for each couple from a growing list of available tests, is becoming an impractical task.

The transition to high throughput technology-based screening will most probably obviate both the mutations' frequency criteria and the ethnicity-dependent recommendations applied today, as was also acknowledged in the new ACMG policy [10]. In this study, we provide proof-of-concept for combining high-throughput technologies for targeting and sequencing a set of hundreds known mutations, tailored to a specific population, to be use in pre-conception carrier screening.

\section{Methods}

Target selection and compiling a mutation panel set We compiled an updated comprehensive list of diseasecausing mutations in the Jewish population from data provided by the Department of Community Genetics at the Israeli Ministry of Health (as of Oct 2011). In the targeted panel we included mutations for Autosomal recessive (AR) or X -linked disorders, in which genetic testing can identify couples at risk, and is likely to influence parental decisions regarding the pregnancy or the clinical management of the newborn.

The online resources used for clinical evaluation included the booklet "Mendelian disorders among Jews" from the MOH website [8], "OMIM" [11] and "GeneReviews" at the NCBI website [12] Human Gene Mutation Database (HGMD) [13], and relevant literature.

More than half of the mutations in the panel were described in non-Ashkenazi patients from various countries of origin. This set of mutations better reflects the current Israeli population, where Jews of

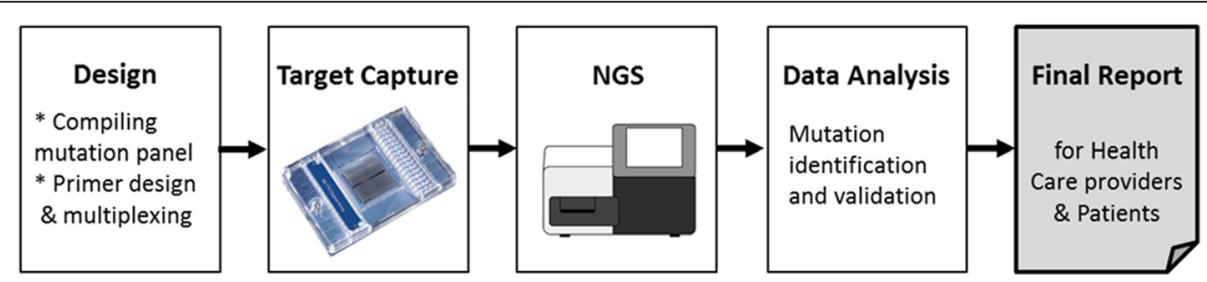

Fig. 1 The general workflow of this study which contains four principal steps: I. Data acquisition and design - creating the panel of mutations to be targeted and designing specific primers and their multiplexing, II. Experimental procedures for target capture (using Fluidigm microfluidics device) and sequencing (MiSeq platform), III. Bioinformatics analysis, mutation identification and validation and IV. Providing final report with the mutations identified in each sample 
non-Ashkenazi origin comprise more than half of the Jewish population (personal communication from the Israeli $\mathrm{MOH})$.

While some of the listed mutations have clear support for being founder mutations (i.e. were identified in several unrelated families), a large proportion were found only in single families, and are currently regarded as "private" mutations. However, we could not exclude the possibility that they do exist (with lower frequencies) in other individuals, and therefore we included them in the panel. The final panel comprised of 370 mutations of both single nucleotide variants (SNVs) and small insertions and deletions (indels) in 148 genes associated with 120 disorders (Additional file 1).

Two of the mutations were substitutions of the same genomic nucleotide to different alternative alleles (both resulting in pathogenic mutations): p. R261P and p.R261Q in the $P A H$ gene and p.P339H and c.1016insC in the $C Y B B$ gene. For the analysis of coverage depth, each of the pairs were counted as one thus in total we had to target 368 genomic positions for 370 mutations.

We also included in the panel four mutations of largerearrangements (deletion or insertion of up to tens of $\mathrm{Kb})$, with characterized genomic breakpoints, but these were eventually excluded from the final analysis. The genomic positions of all mutations were verified, including the precise position of breakpoints in gross insertions and deletions.

\section{Primer design and multiplexing}

To capture the genomic positions of the mutation list, 345 primer-pairs were designed and multiplexed into 45 sub-groups containing 1-12 primer pairs in each pool using Fluidigm custom primer design service. To test the ability to capture and sequence large rearrangements, four primers-pairs were designed to span the breakpoints of four mutations of large deletions or insertions. These primers were expected to capture the abnormal chimeric products that exist only in carriers' genomes (See examples in Additional file 2).

The primers multiplex algorithm (Fluidigm Co.) evaluated the primer-primer interactions in order to minimize the generation of undesirable PCR products, but to maximize PCR specificity and efficiency. The major considerations were to avoid combining primer pairs that produce PCR products that overlap or are separated by less than $5 \mathrm{~kb}$, have high variability of GC content or are not within $20 \%$ of the average size. Amplicon lengths were between 64 and 200 bps, with one outlier amplicon of $418 \mathrm{bp}$ designed for a large deletion mutation (6.7 kb deletion in PAH gene). 54 mutations were designed to be capture by multiple primer pairs, and 49 amplicons covered multiple mutations.

\section{DNA samples}

The study was approved by the ethics committee of Sheba Medical Center, Israel.

We obtained genomic DNA samples from 43 healthy anonymous carriers of various genetic disorders. These carriers were previously identified by traditional genetic assays, and were used as positive controls to examine the method. We included eight biological replicates (different samples that carry the same mutation) and four technical replicates (the same sample tagged with two different barcodes). The control samples were chosen to include all types of genetic variations: 34 single nucleotide substitutions (SNVs), 16 small indels, and five gross re-arrangements, all analyzed in a blinded manner (Table 1, "Knowns").

\section{Target capturing, library preparation and high-throughput sequencing}

Genomic DNA templates were amplified using the 48.48 Access Array IFC, according to the manufacturer's instructions (Fluidigm). Briefly, 48 DNA samples were combined with the 48 multiplex primers pairs on a microfluidic IFC-chip. The IFC-chip was loaded with PCR reagents using the Access Array pre-PCR controller loading script and upon completion it was transferred to a thermocycler. After amplification, pooled amplicons from each of the 48 DNA templates were harvested using Access Array post-PCR controller harvesting script, and used as input for the subsequent off-chip PCR reactions, in which each mini-library underwent tagging with sample specific barcodes and attachment of sequencing platform-specific adapters. The output libraries were purified using Qiagen PCR purification kit and analyzed on an Agilent DNA1000 BioAnalyzer chip. The purified combined libraries were sequenced using MiSeq 150 bps pair-end protocol, according to the manufacturers' standard instructions.

\section{Bioinformatics analysis}

MiSeq sequencing data was de-barcoded to samples by the Illumina BaseSpace server.

Alignments to the human genome (hg19) were performed with BWA version 0.7.4 ${ }^{16}$, applying the mem argument. Depth calculations per target and sample were done with the DepthOfCoverage tool of the GATK toolkit [14].

Three samples were excluded from further analysis of variant calling and depth of coverage statistics: sample 2 and sample 27 had the lowest reads counts (35,142 and 118 reads, less than $1 \%$ of reads in each of the other samples), pointing to poor DNA quality or technical pipetting problem during library preparation; sample 36 was excluded due to insufficient coverage of large number of targets (44 targets with less than 10X), possibly 
Table 1 Summary of all mutations identified in the experiment

\begin{tabular}{|c|c|c|c|}
\hline \multicolumn{4}{|c|}{ "Knowns" } \\
\hline Sample & Gene & Mutation & Detected \\
\hline S3 & HEXA & c.1278insTATC & $\sqrt{ }$ \\
\hline S6 & CFTR & p.F508 del & $\sqrt{ }$ \\
\hline S7 & DYSF & c.1624delG & $\sqrt{ }$ \\
\hline S8 & FANCA & c. $2172-2173+G$ & $\sqrt{ }$ \\
\hline S9 & GBA & p.84dupG & $\sqrt{ }$ \\
\hline $\mathrm{S} 10$ & GJB2 & c.167DelT & $\sqrt{ }$ \\
\hline $\mathrm{S} 10$ & CFTR & p.N1303K & $\sqrt{ }$ \\
\hline S11 & GJB2 & c.35delG & $\sqrt{ }$ \\
\hline S11 & $\mathrm{PAH}$ & A403V & $\sqrt{ }$ \\
\hline S11 & ATM & p.103C > T & $\sqrt{ }$ \\
\hline $\mathrm{S} 12$ & GJB2 & 51_62del12ins1 & $\sqrt{ }$ \\
\hline $\mathrm{S} 13$ & HEXA & c.1278insTATC & $\sqrt{ }$ \\
\hline $\mathrm{S} 13$ & SMARCAL1 & IVS4 -2 A > G & $\sqrt{ }$ \\
\hline S14 & HEXA & p.F304/305del & $\sqrt{ }$ \\
\hline S15 & SPMD1 & p.R610del & $\sqrt{ }$ \\
\hline S15 & PEX6 & p.A809V & $\sqrt{ }$ \\
\hline S16 & $\mathrm{BCKDHB}$ & p.R183P & $\sqrt{ }$ \\
\hline S17 & CERKL & $\operatorname{IVS} 1+1 \mathrm{G}>\mathrm{A}$ & $\sqrt{ }$ \\
\hline $\mathrm{S} 18$ & CFTR & $C .405+1 G>A$ & $\sqrt{ }$ \\
\hline S19 & CFTR & p.G542X & $\sqrt{ }$ \\
\hline S20 & CFTR & p.G85E & $\sqrt{ }$ \\
\hline S21 & CFTR & p.W1282X & $\sqrt{ }$ \\
\hline S22 & FANCC & IVS4 + 4A > T & $\sqrt{ }$ \\
\hline S23 & G6PC & p.R83C & $\sqrt{ }$ \\
\hline S24 & GBA & c. $115+1 G>A$ & $x^{a}$ \\
\hline S25 & GBA & p.N370s & $\sqrt{ }$ \\
\hline S25 & GBA & p.V394L & $\sqrt{ }$ \\
\hline S25 & PEX6 & p.A809V & $\sqrt{ }$ \\
\hline S26 & GBA & p.R496H & $x$ \\
\hline S28 & CFTR & p.Q359K + p.T360K & $\sqrt{ }$ \\
\hline S30 & IKBKAP & c. $2204+6 \mathrm{~T}>\mathrm{C}$ & $\sqrt{ }$ \\
\hline S31 & HEXA & p.G269S & $\sqrt{ }$ \\
\hline S32 & TMEM216 & p.R73L & $\sqrt{ }$ \\
\hline S33 & HEXA & IVS12 + 1G >C & $\sqrt{ }$ \\
\hline S34 & HEXA & p.R170Q & $\sqrt{ }$ \\
\hline S35 & SPMD1 & p.L302P & $\sqrt{ }$ \\
\hline S38 & SPMD1 & p.R496L & $\sqrt{ }$ \\
\hline S39 & HEXA & p.G250V & $\sqrt{ }$ \\
\hline$\$ 40$ & ATM & p.103C > T & $\sqrt{ }$ \\
\hline S41 & CFTR & p.N1303K & $\sqrt{ }$ \\
\hline$\$ 42$ & GJB2 & c.167DelT & $\sqrt{ }$ \\
\hline$\$ 42$ & CFTR & p.N1303K & $\sqrt{ }$ \\
\hline$\$ 43$ & HEXA & c.1278insTATC & $\sqrt{ }$ \\
\hline
\end{tabular}


Table 1 Summary of all mutations identified in the experiment (Continued)

\begin{tabular}{llll}
\hline S44 & DYSF & c.1624delG & $\sqrt{ }$ \\
S45 & GJB2 & 51_62del12ins1 & $\sqrt{ }$ \\
S46 & CFTR & p.N1303K & $\sqrt{ }$ \\
S47 & GJB2 & c.35delG & $\sqrt{ }$ \\
S47 & P.A403V & $\sqrt{ }$ \\
S47 & PAH & p.103C $>$ T & $\sqrt{ }$ \\
S48 & ATM & p.Y1092X & $\sqrt{ }$
\end{tabular}

Large Rearrangements

\begin{tabular}{|c|c|c|c|}
\hline S1 & GALT & Del 5Kb & $\sqrt{ }^{\mathrm{b}}$ \\
\hline S4 & $\mathrm{PAH}$ & Del 6.7Kb & $\sqrt{ }^{\mathrm{b}}$ \\
\hline S3 & MAK & Ins353bp & $\sqrt{ }^{\mathrm{b}}$ \\
\hline$\$ 29$ & MAK & Ins353bp & $\sqrt{ }^{\mathrm{b}}$ \\
\hline S32 & MAK & Ins353bp & $\sqrt{ }^{\mathrm{b}}$ \\
\hline \multicolumn{4}{|c|}{ "Unknowns" } \\
\hline Sample & Gene & Mutation & validated \\
\hline S3 & CLRN1 & p.N48K & $\sqrt{ }$ \\
\hline S3 & ASPA & p.E285A & $\sqrt{ }$ \\
\hline S7 & AMN & c. $208-2 A>G$ & $\sqrt{ }$ \\
\hline S17 & SAMD9 & p.R344X & $\sqrt{ }$ \\
\hline S18 & GUCY2D & c.389delC & NA \\
\hline $\mathrm{S} 18$ & SERPINA1 & p.E342K & $\sqrt{ }$ \\
\hline $\mathrm{S} 18$ & SERPINA1 & p.E264V & $\sqrt{ }$ \\
\hline S22 & GJB2 & p.V37l & $\sqrt{ }$ \\
\hline S31 & $\mathrm{ABCC} 8$ & c.3989-9G > A & $\sqrt{ }$ \\
\hline S31 & PEX6 & p.A809V & $\sqrt{ }$ \\
\hline S33 & SPMD1 & p.R496L & $\sqrt{ }$ \\
\hline S39 & FANCC & IVS4 + 4A > T & $\sqrt{ }$ \\
\hline$\$ 40$ & CFTR & p.L997F & NA \\
\hline$\$ 40$ & FAM161A & c.1355_6delCA & $\sqrt{ }$ \\
\hline S44 & EYS & p. Thr135LeuX25 & $\sqrt{ }$ \\
\hline \multicolumn{4}{|c|}{ Variants with high incident } \\
\hline Gene & mutation & \multicolumn{2}{|l|}{ samples } \\
\hline TRMU & p.A10S & \multicolumn{2}{|c|}{$\mathrm{S} 10, \mathrm{~S} 11, \mathrm{~S} 16, \mathrm{~S} 33, \mathrm{~S} 38, \mathrm{~S} 42, \mathrm{~S} 44, \mathrm{~S} 47$} \\
\hline ACADS & p.G185S & \multicolumn{2}{|c|}{$\mathrm{S} 2, \mathrm{~S} 4^{\mathrm{c}}, \mathrm{S} 8, \mathrm{~S} 12^{\mathrm{c}}, \mathrm{S} 13, \mathrm{~S} 51, \mathrm{~S} 18^{\mathrm{c}}, \mathrm{S} 19, \mathrm{~S} 21, \mathrm{~S} 22, \mathrm{~S} 23^{\mathrm{c}}, \mathrm{S} 24, \mathrm{~S} 26, \mathrm{~S} 28, \mathrm{~S} 33, \mathrm{~S} 35, \mathrm{~S} 36^{\mathrm{c}}, \mathrm{S} 39, \mathrm{~S} 41, \mathrm{~S} 44, \mathrm{~S} 45^{\mathrm{c}}, \mathrm{S} 46, \mathrm{~S} 48$} \\
\hline MYOC & p.R76K & \multicolumn{2}{|c|}{$\mathrm{S} 11, \mathrm{~S} 12, \mathrm{~S} 13, \mathrm{~S} 16, \mathrm{~S} 18, \mathrm{~S} 24, \mathrm{~S} 28, \mathrm{~S} 29, \mathrm{~S} 31, \mathrm{~S} 45, \mathrm{~S} 47$} \\
\hline LIPA & p.G5R & \multicolumn{2}{|c|}{$\mathrm{S} 2, \mathrm{~S} 5^{\mathrm{C}}, \mathrm{S} 6^{\mathrm{C}}, \mathrm{S} 29, \mathrm{~S} 37, \mathrm{~S} 44, \mathrm{~S} 48$} \\
\hline
\end{tabular}

Ideantified manually with $26 \%$ mutant allele

bdetected by finding exact match of chimeras sequences (explained in the Methods)

cfound as homozygous

due to technical microfluidics malfunction in specific wells of the Access Array.

Single nucleotide variants (SNVs) and small indels were identified with the GATK toolkit version 3.1.1 with default parameters (including realignment and recalibration steps) [14]. Variants detected in technical replicates were mostly identical, and almost all discordant ones were in poorly covered positions (DP $<10)$ in one of the duplicates. Variant Call Format (VCF) files were then intersected with a list of positions of the mutations of interest. For small insertions or deletions, a search within adjacent genomic intervals was performed, and positions were manually curated as needed. 
Variants detection criteria were minimal depth of coverage (DP) of at least 50X and mutant allele percentage between 30 and $70 \%$ for calling heterozygotes.

Mutations of large re-arrangements were detected by identifying reads that align to mutant (chimeric) junction reference sequence (could be captured only in samples of carriers, see Additional file 2).

\section{Validation of mutations}

Novel and unexpected mutations that were identified in the experiments (i.e. not previously known to be present in the samples) were validated by Sanger sequencing in ABI PRISM 3730xl Genetic Analyzer (Applied Biosystems, Warrington, UK).

\section{Results}

\section{Target Enrichment Performance}

Forty-eight DNA samples were captured for 368 genomic positions (of 370 mutations) using the combined high-throughput methods of microfluidics based multiplex PCR and NGS.

Sequencing the captured library with Illumina's MiSeq yielded a total of $5.4 \mathrm{~Gb}$ with quality score of $\geq$ Q30 (i.e. sequencing error probability of 1 in 1000) in $92.1 \%$ of bases. 33,241,984 reads could be assigned to specific samples by their barcodes. The average reads count was 692,541 per sample (range 392,815-1,455,638, excluding two samples, see methods for details).

Average alignment rate to the human genome was $99.8 \%$ per sample (range $96-100 \%$ ), with $95 \%$ of reads mapping to the expected amplicons, indicating high specificity of the primer design and capture procedure. 365 of 368 (99 \%) genomic positions of the targeted mutations were covered by at least 1 read, indicating successful target capture by nearly all primer-pairs.

As the purpose of the experiment is to detect heterozygote carriers of these mutations, sufficient coverage at the mutation genomic position must be achieved. After excluding three samples due to technical problems (see Methods), the sensitivity of the capture protocol was very high, as 358 of 368 (97\%) of the targeted genomic positions had an average depth per sample of at least 150X, with more than 50X in all samples (Fig. 2).

The target capture with the multiplexed microfluidics PCR technology enabled to essentially perform 16,650 independent assays in this experiment (where "assay" defined as determining the presence of a single mutation in a specific sample)., Reassuringly, the average depth of an assay in our experiment was $2511 \mathrm{X}$, with $98 \%$ of the assays covered by at least 50 - reads. The depth of coverage of most targets was uniform across the samples (Fig. 3).

From the tested individuals (single samples) aspect, an effective and well-designed high-throughput carrier test should have minimal inconclusive results, i.e. number of mutations that cannot be confidently identified due to low coverage (under a pre-defined threshold). Excluding the technically failed samples (see Methods), the minimal percentage of conclusive tests, with DP threshold of at least 50X in a sample, was $98 \%(360 / 368)$ showing high completeness also per each sample analyzed.

Of note, most of the low covered targets were consistent across samples, indicating that better primer design might increase their capture efficiency.

\section{Variant detection}

To assess the ultimate goal of this protocol -its capability to detect the mutations of interest- we used 40 samples of known heterozygote of 50 different mutations (both SNVs and indels). These samples were previously identified by traditional methods routinely applied for carrier screening.

The samples were blindly screened using GATK variant calling pipeline, and 48 (96\%) of these mutations

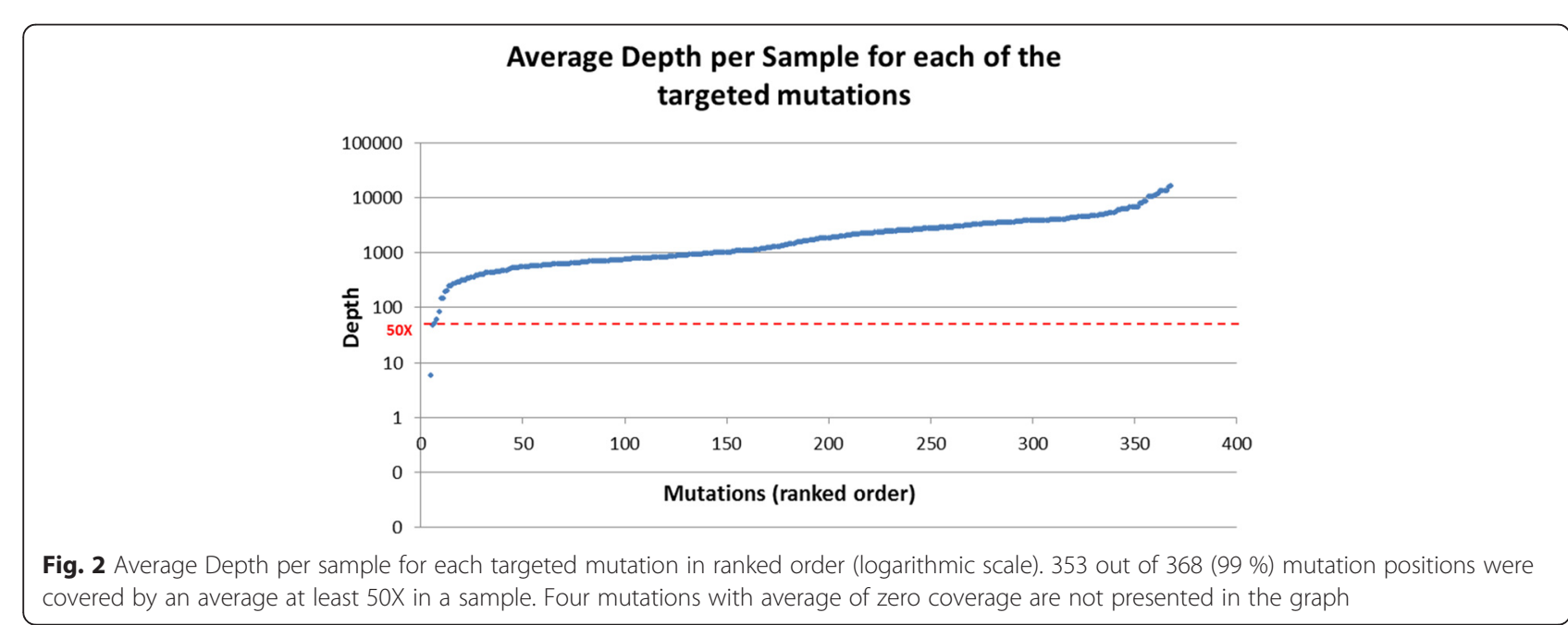




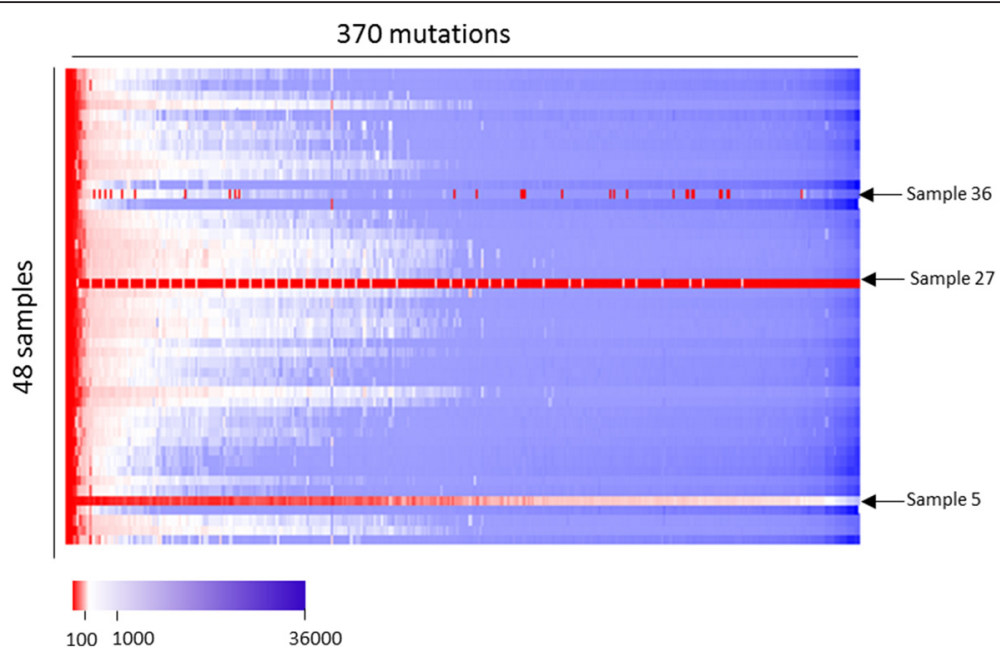

Fig. 3 Heat map showing the depth of coverage of each mutation in each of the samples. A matrix of 17760 assays (368 mutation positions X 48 samples) is presented with color coded of red for less than 100X (our defined threshold) and white to purple gradient for more than 100X from low to high coverage, respectively. The mutations are presented in ranked order by their average sample depth. Marked with arrows are the three samples that were excluded from analysis due to technical problems (see Methods)

were successfully called in their expected samples and in heterozygotes state (Table 1, "Knowns"). The two false negatives were mutations in the GBA gene $(\mathrm{R} 496 \mathrm{H}$ and c. $115+1 G>A$ ), causing Gaucher disease. Identifying mutations in this gene has been recognized as problematic due to the presence of the paralogues pseudogene GBAP1 $[15,16]$. The high similarity between GBA and GBAP1 pose difficulties in specific capture and accurate mapping of reads, and challenges mutation detection, especially where GBAP1 variants can be confused with GBA mutations [17]. For example, in the c. $115+1 \mathrm{G}>\mathrm{A}$ mutation, the mutated allele $(\mathrm{A})$ corresponds to the actual normal reference sequence of the pseudogene, so that reads originated from the GBAP1 can be identical to ones derived from carrier with mutated GBA. Manual inspection for this mutation had revealed that the mutant allele is present with allele frequency of $26 \%$ at the sample of a known carrier, as expected if the reads from GBA and GBAP1 were captured in even ratio.

Nevertheless, we correctly identified other mutations in this gene (e.g.: p.84dupG in sample 9, p.N370S and V394L in sample 25) captured by other amplicons, proving that with careful primer design, adjusting read length and ascertainment of phasing during the bioinformatics analysis, mutations in such genes can be identified.

In addition to the expected mutations described above, we detected 64 additional variants from the target list that were not previously identified in the samples (Table 1,"Unknowns"). 49 of these were accounted for by four substitutions, each identified in multiple samples: p.G5R in LIPA.p.A10S in TRMU, p.G5R in LIPA p. R76K in MYOC and p.G185S in ACADS which were identified in 7,8,11 and 23 samples respectively; in some samples they were called as homozygous (Table 1,"Unknowns - variants with high incident"). These observations support their re-classification as common and benign polymorphisms, and we suggest that they should not be included in future versions of the mutation panel. The other 15 unexpected mutations were found each in a single sample; 13 out of 13 were validated by Sanger sequencing or other methods.

In total, we were able to validate $61 / 63(97 \%)$ of the mutations that were identified in the experiment. The Average DP in these mutations was 519X and the average percentage of mutant allele was $49 \%$ (range 36$61 \%$, Additional file 3).

Finally, we also tested the ability of the capture protocol to identify mutations of large re-arrangements with known breakpoints, using five samples of carriers and special designed primers. We successfully identify reads that aligned to mutant junction reference sequences in those samples, proving that such mutations type could also be included in the panel (see examples in Additional file 2). However, as these mutations were called with insufficient depth, it requires further calibration.

\section{Discussion}

We explored the feasibility of coupling microfluidics technology for target capture with NGS as a fast and cost-effective method for performing prenatal carrier screening of the Israeli Jewish population. Our protocol enabled performing $\sim 17,000$ assays in single experiment, in each we aimed to determine the presence of a specific mutation in a specific sample. The results of the pilot study presented here were promising in both aspects of 
target capture performance and the accuracy of mutation detection.

The capture efficiency was very high as $97 \%$ all of the targeted mutation' positions were captured with at least $50 \mathrm{X}$ in all samples (excluding the three faulty samples). The sensitivity, measured as the percentage of mutations that were covered in adequate depth (50X) to enable reliable variant identification, was $98 \%$ both per individual sample tested and globally, i.e. the total assays produced on the chip. This minimizes the need to repeat samples or perform supplemental analysis by other methods such as Sanger sequencing. Finally and most importantly, we were able to detect $96 \%$ all mutations present in the positive control set, and validate all the newly discovered ones in the validation set.

One of the major advantages of the microfluidics device is the lack of potential cross hybridization, which was reflected in the very low proportion of off-target reads (high specificity). Another advantage of this target capture technology is its dynamism and flexibility. Unlike fixed genotyping methods, such as array-based hybridization probes [18], the panel set can be easily updated by changing the composition of primers in the microfluidics device. New mutations are constantly been discovered, and the panel can be adapted to keep pace with these findings.

Undoubtedly, NGS is becoming the method of choice for performing genetic testing in a clinical setting. Other studies utilizing NGS for carrier screening for AR diseases have been published. Bell et al. captured and sequenced a subset of the exome (containing only genes that were previously associated with AR diseases) [19] and Umbarger et al. focused on sequencing the entire coding region of 15 genes [20]. In contrast, we intentionally focused on a specific set of mutations. In our view, although the cost of sequencing entire exomes or genomes is constantly dropping, there are several advantages in using a targeted approach.

First, in large population screening, such as prenatal carrier screening in Israel, tens of thousands of samples are tested per year, and results must be obtained automatically and in a timely fashion. For reliable variant calling that meets clinical standards, sequencing data of both high quality and depth is required. Achieving these parameters in large genomic regions (such as exomes or genomes) and in large population samples is not yet cost-effective. Apart from the sequencing itself, there are also costs involving the computational burden of analyzing and storing large data sets, providing additional advantage to approaches that generate a relatively low and manageable amount of data.

Second, the fine-targeted approach is highly suitable for screening genetically homogenous or isolated populations, such as the Jewish individuals in Israel, in which known mutations in specific genes account for the majority of the carriers [21, 22].

Third and most fundamental, is the concern regarding ambiguous results. In any method that is not constrained to identifying already known mutations, the possibility of identifying variants with uncertain significance (VUS) is inevitable. Determining the biological and clinical significance of such variants is in many instances difficult and may yield inconclusive results. Not every presumed loss-of-function variant has diseasecausing potential even in well annotated Mendelian genes, such as CFTR [23] or HEXA [24]. Moreover, elucidating VUS requires a personalized approach to data interpretation, which is beyond the primary purpose of large population screening, and may impose major difficulties for healthcare providers that have to convey the genetic information to the prospective parents. While broader-scaled sequencing is advantageous in a research setting, or in clinical testing of affected individuals, it still lacks sufficient clinical validity and utility for screening healthy individuals. In our view, clinical screening should identify only variants that are unequivocally disease associated and whose detection in carrier screening is expected to aid in reproductive decisions or lead to early intervention. In their recent policy statement on expanding prenatal/preconception carrier screening with high-throughput technologies, the American College of Medical Genetics has raised these concerns and stated that the tests should "include specific citations that support inclusion of the mutations for which screening is being performed" [10].

For these reasons, we suggest that for the purpose of pre-conception screening, a fine targeted method is necessary, and have included in our panel only mutations that were previously reported to be disease causing.

In this mini-cohort of 48 samples, we have identified four variants that are unlikely to be associated with any pathology. When compiling the mutation panel set, these variants were included because previous publications described them as disease-associated mutations. Two variants (p.A10S in TRMU and.G185S in ACADS) also appear in HGMD [13], and are flagged as "clinically associated" in dbSNP, p.R76K in MYOC was believed to contribute to glaucoma in a di-genic inheritance [25] and p.G5R in LIPA was shown in a functional assay to cause reduced enzymatic activity [26]. These variants were highly prevalent in our sample set, were identified as homozygous in some of the samples, and are also present in 1000 Genomes with minor allele frequency of at least $10 \%$. Therefore, we suggest that these variants should be re-evaluated and considered as benign polymorphisms, and be excluded from future panels. Similar findings in other reported mutations were also observed by Bell et al [19], highlighting the need to improve 
databases of clinically associated variants. Nevertheless, these findings illustrate the strength of our screening protocol. By simultaneously screening dozens of samples for each of the mutations, rapid information about the frequencies of all tested mutations will accumulate, and this in turn can help to correct such misinterpretations and to optimize screening recommendations.

Before implementing our protocol into clinical care, a few improvements are required. First, mutations and disorders included should be updated to be compatible with the latest professional guidelines and databases. Second, for the poorly covered mutations and the large rearrangements primers should be re-designed. Third, redundant amplicons for each mutation can be introduced to minimize technical capture failures. Fourth, the bioinformatics analysis for mutation detection should be finetuned, especially in challenging genomic regions such as genes which have pseudogenes. In addition, In light of the excessive depth achieved in our experiment for some of the mutations, further multiplexing of primers for additional mutations and/or increasing the number of samples in the parallel sequencing can be considered, to further reduce the cost per sample and per mutation.

\section{Conclusions}

This is a proof-of-concept study designed to perform large pre-conception population screening for thousands of genetic tests in single experiment, without compromising on high confident of variant detection.

Our experimental system is practical and costeffective, balancing the high-throughput potential of the latest cutting-edge technologies and the evidence-based knowledge that is crucial in a clinical setting. The current version of the protocol can be implemented into a clinical service after few modifications. Moreover, this approach can be also be customized for screening any other populations with different mutation profile.

\section{Additional files}

Additional file 1: List of mutations included in the screening panel.

Additional file 2: Examples of primer design and supporting reads for the large-rearranged mutations. A. Deletion of $\sim 5 \mathrm{~Kb}$ in the GALT gene, leading to Galactosemia. This mutation is composed of four breakpoints, leading to two large deletions and one small insertion, and resulting in the loss of almost the entire gene (adapted from Coffee et al. [27]). Vertical arrows depict the breakpoints, and horizontal arrows mark the primers used for capture. Primers $1 F+1 R$ are used to capture the amplicon created in the $5^{\prime}$ deleted region, and the $2 \mathrm{~F}+2 \mathrm{R}$ primers are used to capture the amplicon created in the $3^{\prime}$ indel region. B. Insertion of a $353 \mathrm{bp}$ Alu element into the MAK gene leads to Retinitis Pigmentosa (found by Tucker et al. [28]). (PNG $69 \mathrm{~kb}$ )

Additional file 3: Total depth and percentage of mutant reads in the validated heterozygote mutations (both previously knowns and unknowns). As expected, the percentage of mutant alleles approximated $50 \%$ for both SNPs and indels. (PNG $17 \mathrm{~kb}$ )

\section{Competing interests}

$\mathrm{ML}$ and $\mathrm{ML}$ were employees of Fluidigm Corporation. Fluidigm LTD. contributed to this manuscript by providing reagents, primer design, and multiplex pooling strategy.

Fluidigm LTD was not involved in any part of the experimental procedures, data analysis and conclusions.

\section{Authors' contributions}

MG conceived the study, participated in the research design and its coordination, analyzed and interpreted the data, and drafted the manuscript. KK participated in the protocol design, and carried out the library preparation with the access array, contributed to data interpretation and drafting the manuscript, MB performed the sequence alignment, and took part in the bioinformatics analysis, $M L, M L$ performed the primers design and multiplexing, HL,HR,EP provided samples and performed part of the validations of the mutations. EP and EYL participated in the research design. EYL conceived the study, and reviewed the results. All authors have read and approve of the final version of the manuscript.

\section{Acknowledgements}

We thank Joel Zlotogora, Department of Genetic Community at the Israeli Ministry of Health, for providing us with the updated database of mutations in the Jewish population and for fruitful discussion, Gideon Rechavi, Sheba Medical Center for the assistance with the experimental system, Anat Blumenfeld from the Department of Ophthalmology, Hadassah Hebrew University Medical Center, Jerusalem for helping with validation of several of the mutations, llana Buchumenski for her assistance with figures formation and Nurit Gal-Mark and Lily Bazak, for reviewing the manuscript and helpful suggestions.

This work was supported by the I-CORE Program of the Planning and Budgeting Committee and the Israel Science Foundation (grant nos. 41/11).

\section{Author details}

${ }^{1}$ The Mina and Everard Goodman Faculty of Life Sciences, Bar-llan University, Ramat Gan 52900, Israel. Fluidigm corporation, South San Francisco,

California. ${ }^{3}$ The Danek Gertner Institute of Human Genetics, Sheba Medical

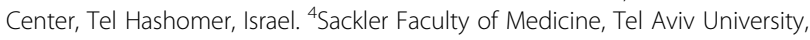
Tel Aviv, Israel.

Received: 14 June 2015 Accepted: 2 May 2016

Published online: 13 May 2016

\section{References}

1. Sweetman L. Newborn screening by tandem mass spectrometry: gaining experience. Clin Chem. 2001;47(11):1937-8.

2. Kaback MM. Population-based genetic screening for reproductive counseling: the Tay-Sachs disease model. Eur J Pediatr. 2000;159 Suppl 3:S192-5.

3. Kronn D, Jansen V, Ostrer H. Carrier screening for cystic fibrosis, Gaucher disease, and Tay-Sachs disease in the Ashkenazi Jewish population: the first 1000 cases at New York University Medical Center, New York, NY. Arch Intern Med. 1998;158(7):777-81.

4. Scott SA, Edelmann L, Liu L, Luo M, Desnick RJ, Kornreich R. Experience with carrier screening and prenatal diagnosis for 16 Ashkenazi Jewish genetic diseases. Hum Mutat. 2010;31(11):1240-50.

5. Ferreira JCP, Schreiber-Agus N, Carter SM, Klugman S, Gregg AR, Gross SJ. Carrier testing for Ashkenazi Jewish disorders in the prenatal setting: navigating the genetic maze. Am J Obstet Gynecol. 2014;211(3):197-204.

6. Zlotogora J, van Baal S, Patrinos GP. Documentation of inherited disorders and mutation frequencies in the different religious communities in Israel in the Israeli National Genetic Database. Hum Mutat. 2007;28(10):944-9.

7. Israeli Society of Medical Genetics (ISMG). Genetic screening tests for the general population. [Online]. Available: http://e-med.co.il/ismg/tests_general. asp. Accessed 01 May 2015.

8. Israeli Ministry Of Health. Recommendations for Genetic Screening. [Online] Available: http://www.health.gov.il/Subjects/Genetics/checks/Pages/ GeneticTestingRecommendations.aspx. Accessed 01 May 2015.

9. Cohen T, Vardi-Saliternik R, Friedlander Y. Consanguinity, intracommunity and intercommunity marriages in a population sample of Israeli Jews. Ann Hum Biol. 2004;31(1):38-48. 
10. Grody WW, Thompson BH, Gregg AR, Bean LH, Monaghan KG, Schneider A, Lebo RV. ACMG position statement on prenatal/preconception expanded carrier screening. Genet Med. 2013;15(6):482-3.

11. Baltimore: and 1996 hopkins University, Center for Medical Genetics. OMIM (online Mendelian inheritance in man. [Online]. Available: http://www.ncbi. nIm.nih.gov/omim. Accessed June 2015.

12. Pagon RA. In: Adam MP, Ardinger HH, Wallace SE, Amemiya A, Bean LJH, Bird TD, Fong C-T, Mefford HC, Smith RJH, Stephens K. GeneReviews. Seattle (WA): University of Washington, Seattle; 1993-2016. http://www.ncbinlm.nih. gov/books/NBK1116/.

13. Stenson PD, Ball EV, Mort M, Phillips AD, Shiel JA, Thomas NST, Abeysinghe S, Krawczak M, Cooper DN. Human Gene Mutation Database (HGMD): 2003 update. Hum Mutat. 2003;21(6):577-81.

14. McKenna A, Hanna M, Banks E, Sivachenko A, Cibulskis K, Kernytsky A, Garimella K, Altshuler D, Gabriel S, Daly M,DePristo MA. The genome analysis toolkit: a MapReduce framework for analyzing next-generation DNA sequencing data. Genome Res. 2010;20(9):1297-303.

15. Brown JT, Lahey C, Laosinchai-Wolf W, Hadd AG. Polymorphisms in the glucocerebrosidase gene and pseudogene urge caution in clinical analysis of Gaucher disease allele c.1448T > C (L444P). BMC Med Genet. 2006;7:69.

16. Wafaei JR, Choy FYM. Glucocerebrosidase recombinant allele: molecular evolution of the glucocerebrosidase gene and pseudogene in primates. Blood Cells Mol Dis. 2005;35(2):277-85.

17. Martínez-Arias R, Comas D, Mateu E, Bertranpetit J. Glucocerebrosidase pseudogene variation and Gaucher disease: Recognizing pseudogene tracts in GBA alleles. Hum Mutat. 2001:17(3):191-8.

18. Pastinen T, Perola M, Ignatius J, Sabatti C, Tainola P, Levander M, Syvänen AC, Peltonen L. Dissecting a population genome for targeted screening of disease mutations. Hum Mol Genet. 2001;10(26):2961-72.

19. Bell CJ, Dinwiddie DL, Miller NA, Hateley SL, Ganusova EE, Mudge J, Langley RJ, Zhang L, Lee CC, Schilkey FD, Sheth V, Woodward JE, Peckham HE, Schroth GP, Kim RW, Kingsmore SF. Carrier testing for severe childhood recessive diseases by next-generation sequencing. Sci Transl Med. 2011;3(65):65ra4

20. Umbarger MA, Kennedy CJ, Saunders P, Breton B, Chennagiri N, Emhoff J, Greger V, Hallam S, Maganzini D, Micale C, Nizzari MM, Towne CF, Church GM, Porreca GJ. Next-generation carrier screening. Genet Med. 2014;16(2):132-40.

21. Zlotogora J, Bach G, Munnich A. Molecular basis of mendelian disorders among Jews. Mol Genet Metab. 2000;69(3):169-80.

22. Rosner G, Rosner S, Orr-Urtreger A. Genetic testing in Israel: an overview. Annu Rev Genomics Hum Genet. 2009;10:175-92.

23. Ridge $P G$, Miller C, Bayrak-Toydemir P, Best DH, Mao R, Swensen JJ, Lyon E, Voelkerding KV. Cystic fibrosis testing in a referral laboratory: results and lessons from a six-year period. J Clin Bioinforma. 2013;3(1):3.

24. Hoffman JD, Greger V, Strovel ET, Blitzer MG, Umbarger MA, Kennedy C, Bishop B, Saunders P, Porreca GJ, Schienda J, Davie J, Hallam S, Towne C. Next-generation DNA sequencing of HEXA: a step in the right direction for carrier screening. Mol Genet genomic Med. 2013;1(4):260-8.

25. Geyer O, Wolf A, Levinger E, Harari-Shacham A, Walton DS, Shochat C, Korem S, Bercovich D. Genotype/phenotype correlation in primary congenital glaucoma patients from different ethnic groups of the Israeli population. Am J Ophthalmol. 2011;151(2):263-71.e1.

26. Zschenker O, Jung N, Rethmeier J, Trautwein S, Hertel S, Zeigler M, Ameis D. Characterization of lysosomal acid lipase mutations in the signal peptide and mature polypeptide region causing Wolman disease. J Lipid Res. 2001;42(7):1033-40

27. Coffee B, Hjelm LN, DeLorenzo A, Courtney EM, Yu C, Muralidharan K. Characterization of an unusual deletion of the galactose-1-phosphate uridyl transferase (GALT) gene. Genet Med. 2006;8(10):635-40.

28. Tucker BA, Scheetz TE, Mullins RF, DeLuca AP, Hoffmann JM, Johnston RM, Jacobson SG, Sheffield VC, Stone EM. Exome sequencing and analysis of induced pluripotent stem cells identify the cilia-related gene male germ cell-associated kinase (MAK) as a cause of retinitis pigmentosa. Proc Nat Acad Sci U S A. 2011;108(34):E569-76.

\section{Submit your next manuscript to BioMed Central and we will help you at every step:}

- We accept pre-submission inquiries

- Our selector tool helps you to find the most relevant journal

- We provide round the clock customer support

- Convenient online submission

- Thorough peer review

- Inclusion in PubMed and all major indexing services

- Maximum visibility for your research

Submit your manuscript at www.biomedcentral.com/submit
Biomed Central 